\title{
Evaluation on the phagocytosis of apoptotic spermatogenic cells by Sertoli cells in vitro through detecting lipid droplet formation by Oil Red O staining
}

\author{
Huizhen Wang, Haikun Wang, Weipeng Xiong, Yongmei Chen, Quanhong Ma, Jing Ma, Yehua Ge \\ and Daishu Han \\ Department of Cell Biology, School of Basic Medicine, Peking Union Medical College, Institute of Basic Medical \\ Sciences, Chinese Academy of Medical Sciences, 5 Dong Dan San Tiao, Beijing 100005, People's Republic of China
}

Correspondence should be addressed to D Han; Email: dshan@ibms.pumc.edu.cn

\begin{abstract}
During spermatogenesis, more than half of the differentiating spermatogenic cells undergo apoptosis before they mature into spermatozoa. Ultrastructure studies showed that the formation of lipid droplets in Sertoli cells was associated with phagocytosis of residual bodies and apoptotic germ cells by Sertoli cells. Here, a relationship between the phagocytosis of apoptotic spermatogenic cells and lipid droplet formation in Sertoli cells was studied in vitro by Oil Red O (ORO) staining. The results confirmed that the formation of lipid droplets was a result of phagocytosis of apoptotic spermatogenic cells in Sertoli cells. By comparing phagocytosis of apoptotic spermatogenic cells and thymocytes by Sertoli cells to that by macrophages, we demonstrated that the lipid droplets accumulation in phagocytes depended on phagocytosed apoptotic cell type, but not phagocyte type. However, the size of lipid droplets was related to the type of phagocytes. By this approach, we found that Sertoli cells at different postnatal stages of development had a similar phagocytic ability. These results suggested that the detection of lipid droplets by ORO staining was a practical method to evaluate the phagocytic functions of Sertoli cells in vitro. This approach could also be considered as an in vitro model to study the lipid formation, metabolism, and function in Sertoli cells.

Reproduction (2006) 132 485-492
\end{abstract}

\section{Introduction}

Spermatogenesis is a complex process of cellular differentiation by which a spermatogonial stem cell is gradually transformed into a highly differentiated haploid spermatozoon. In this process, spontaneous apoptotic death of spermatogenic cells was observed during postnatal first wave spermatogenesis and adult life (Allan et al. 1992, Packer et al. 1995, BlancoRodriguez \& Martinez-Garcia 1996). As an intrinsic homeostasis, it had been estimated that $25-75 \%$ expected sperm yield were lost during spermatogenesis (Oakberg 1956, Huckins 1978, Johnson et al. 1983, Dym et al. 1994). In later stage of spermiogenesis, the cytoplasmic portions of elongated spermatids are shed and formed residual bodies during extrusion of differentiated sperm into the lumen of the seminiferous tubule (Kerr \& de Kretser 1974, Chemes 1986, Jegou 1991). As the only somatic cell type in seminiferous epithelium, Sertoli cells play critical roles to endocytose and degrade residual bodies and apoptotic spermatogenic cells
(Russell \& Clermont 1977, Chemes 1986, Pineau 1991, Miething 1992). Although the effect of phagocytosis on testis function has not been intensely analyzed, one report showed that the phagocytic elimination of apoptotic cells was necessary for healthy spermatogenic cells to proceed through spermatogenesis (Ren \& Savill 1998).

In the early years, the evaluations on phagocytosis by Sertoli cells were based on the observations of lysosomes and lipid inclusions in testis section by electron microscope. In rat, the number of lysosomes in Sertoli cells showed cyclic variations during the cycle of the spermatogenesis. A few lysosomes were presented in Sertoli cells at stages I-IV of the seminiferous epithelium and progressively increased to be numerous at stages VI-IX, the time of spermiation and forming residual bodies. After spermiation, the number of lysosomes rapidly decreased (Kerr \& De Kretser 1975, Chemes 1986, Ueno \& Mori 1990). Lipid inclusions in Sertoli cells also showed a cyclic variation based on the stages of seminiferous epithelium (Kerr et al. 1984, Ueno \& 
Mori 1990). However, the timing of cyclic appearance of lipids was different from that of lysosomes. Maximum lipid contents in Sertoli cells appeared at stages IX-XIV of the spermatogenic cycle, then lipid numbers were dramatically declined at stages I-III and remained low level at stages IV-VIII.

In recent years, an in vitro model to investigate the phagocytosis of germ cells by Sertoli cells was established (Shiratsuchi et al. 1997, 1999). Using this method, apoptotic spermatogenic cells labeled with fluorescence were co-cultured with primary isolated Sertoli cells, and the mechanisms of the phagocytosis by Sertoli cells were studied. One problem with this in vitro model was a complication to distinguish germ cells phagocytosed by Sertoli cells from those adhered to the Sertoli cells (Shiratsuchi et al. 1997), which made difficult to evaluate the phagocytic function of Sertoli cells.

Here, we introduce a new in vitro approach that is applicable to evaluate the phagocytosis of apoptotic spermatogenic cells by Sertoli cells through detecting lipid droplets using Oil Red O (ORO) staining. Using this method, we investigated the phagocytic function of Sertoli cells at different developmental stages postnatal, and the lipid formation after the phagocytosis of apoptotic germ cells and thymocytes by Sertoli cells and macrophages respectively.

\section{Materials and Methods}

\section{Animals}

All C57BL/6 mice used in this study were obtained from the Laboratorial Animal Center of Beijing University (Beijing, China) and maintained in a temperature- and humidity-controlled room on a $12 \mathrm{~h}$ light: $12 \mathrm{~h}$ darkness cycle. They had free access to food and water. All the measures taken for the mice were in accordance with approved guidelines (Guideline for the Care and Use of Laboratory Animals) established by the Chinese Council on Animal Care.

\section{Isolation of Sertoli cells and preparation of apoptotic spermatogenic cells}

The procedure for the isolation of Sertoli and spermatogenic cells was based on previous description (Cheng et al. 1986) with a modification. Briefly, mice were anesthetized with $\mathrm{CO}_{2}$ and then killed by cervical dislocation. Decapsulated testes were incubated with $0.5 \mathrm{mg} / \mathrm{ml}$ collagenase (Sigma) at room temperature for $15 \mathrm{~min}$ with gentle oscillation, and then were filtered through $80 \mu \mathrm{m}$ copper meshes to eliminate interstitial cells. Seminiferous tubules were resuspended in the collagenase at room temperature for $20 \mathrm{~min}$ to remove myoid cells. The tubules were then incubated with $1 \mathrm{mg} / \mathrm{ml}$ hyaluronidase (Sigma) for $25 \mathrm{~min}$ with gentle oscillation and pipetting. The cells were washed three times with F12/Dulbecco's modified Eagle's medium (DMEM; Gibco), and plated on culture dish in F12/DMEM supplemented with sodium bicarbonate $(1.2 \mathrm{mg} / \mathrm{ml})$, penicillin $(100 \mathrm{U} / \mathrm{ml})$ and streptomycin $(100 \mu \mathrm{g} / \mathrm{ml})$, and $10 \%$ fetal calf serum (Gibco). The cells were maintained in a humidified atmosphere of 95\% air:5\% $\mathrm{CO}_{2}$ (v:v) at $32{ }^{\circ} \mathrm{C}$ for $48 \mathrm{~h}$. Thereafter, the spermatogenic cells suspending in medium were collected and cultured for another 2 days to induce spontaneous apoptosis as previous description (Shiratsuchi et al. 1997). The Sertoli cells attaching on the dish were treated with a hypotonic solution (20 mM Tris, $\mathrm{pH}$ 7.4) for 2 min to remove the spermatogenic cells adhering to the Sertoli cells. Twenty-four hours later, the Sertoli cells were detached with trypsin and seeded in 24-well tissue-culture plate at $5 \times 10^{4}$ cells/well and cultured for an additional $24 \mathrm{~h}$ for phagocytosis assay. The purity of Sertoli cells was determined by immunofluorescence staining of Wilms' tumor nuclear protein 1 (WT1, a marker of Sertoli cells).

\section{Isolation and culture of macrophages}

The procedure of macrophage preparation was performed based on a previous approach (Chong et al. 2005). Briefly, resident peritoneal macrophages were collected from mouse peritoneal cavities by lavage with $5 \mathrm{ml}$ cold PBS containing 2\% fetal calf serum. The cells were cultured in DMEM supplemented with $10 \%$ fetal calf serum on tissue-culture dishes in a humidified atmosphere of $95 \%$ air: $5 \% \mathrm{CO}_{2}(\mathrm{v}: \mathrm{v})$ at $37^{\circ} \mathrm{C}$. After $2 \mathrm{~h}$, suspending cells were removed by washing with PBS. Adherent cells were then liberated and replated out at $5 \times 10^{4}$ cells/well in a 24 -well culture plate.

\section{Preparation of apoptotic thymocytes}

The protocol for obtaining apoptotic thymocytes was based on a previous method (Scott et al. 2001). Briefly, the thymocytes were harvested from the thymus of 3-week-old C57BL/6 mice, and treated with $2 \mu \mathrm{M}$ dexamethasone (Sigma) for $4 \mathrm{~h}$ to induce apoptosis. The apoptotic cells were washed with PBS, and resuspended in DMEM containing 10\% fetal calf serum for phagocytosis assay.

\section{Phagocytosis of apoptotic cells}

The procedure of phagocytosis of apoptotic cells by Sertoli cells and macrophages was performed based on previous protocol (Shiratsuchi et al. 1997) with modifications. The apoptotic spermatogenic cells or thymocytes $\left(5 \times 10^{5}\right)$ were added to the Sertoli cells or macrophages cultured in 24-well plates. The co-cultures were maintained at $32{ }^{\circ} \mathrm{C}$ for Sertoli cells and $37^{\circ} \mathrm{C}$ for macrophages until ORO staining. Single-cultured Sertoli cells and macrophages were used as controls at each 
assay point. Total 100 phagocytic cells from three repeat wells were analyzed for each point. The mean value was presented in the results.

\section{ORO staining}

The co-cultures were washed in PBS by pipetting for removing suspended apoptotic cells. The phagocytic cells were fixed with $10 \%$ formalin for $40 \mathrm{~min}$. After a wash with PBS, the cells were stained with ORO (Sigma) solution (ORO-saturated solution in isopropanol:water, 3:2) for 15 min as previous description (Lillie \& Fullmer 1976). Then, the cells were washed with $70 \%$ alcohol for $5 \mathrm{~s}$ to remove background staining. Finally, the cells were rinsed in tap water, counterstained with Harris hematoxylin (10 s), and mounted in glycerol-PBS (9:1) for observation.

\section{Morphometric procedures}

The amount of lipid droplets by ORO staining in Sertoli cells was quantified using a microscope image analyzer (Yali'en, Beijing, China). The area of Sertoli cell nucleus was used as a reference to that of lipid droplets. The area ratio of lipid droplets to nucleus in Sertoli cells was used to evaluate quantitatively lipids. The morphometric data were analyzed statistically with Student's $t$-test.

\section{Results}

\section{Lipid droplet formation in Sertoli cells after phagocytosis of apoptotic spermatogenic cells}

Sertoli cell is phagocytic and helps to remove the residual bodies and degenerating germ cells by phagocytosis in vivo. Such activities lead to the formation of lipid droplets that are located throughout the cytoplasm of Sertoli cell. In order to investigate whether the lipid droplets in Sertoli cells could be a criterion to evaluate the phagocytic activities of Sertoli cell in vitro, a study on the time course for the lipid droplet formation during co-culture of primary Sertoli cells and degenerating germ cells was carried out. Sertoli and spermatogenic cells were isolated from the testes of 21-day-old C57BL/6 mice. The purity of Sertoli cells was $95 \%$ based on the immunofluorescence staining of WT1 (data not shown). The spermatogenic cell population was a mixture of germ cells at different differentiation stages. The spermatogenic cells were cultured for $48 \mathrm{~h}$ to remove contaminated somatic cells that adhered to the culture dish and induce apoptosis of the germ cells. The spermatogenic cells with a viability of $80 \%$ as determined by Trypan blue staining were subjected to a phagocytosis assay through co-cultured with the Sertoli cells. In order to determine the time course of the lipid droplet formation, ORO staining was performed at $0,6,12,24$, and $48 \mathrm{~h}$ after co-culture of
Sertoli and spermatogenic cells. At each time point, single-cultured Sertoli cells were stained as negative controls. In order to analyze lipid droplet formation in Sertoli cells in a quantitative manner, the ratios of lipid droplets area to nucleus area of Sertoli cell were determined by image analyzer. The results were presented in Fig. 1. At $0 \mathrm{~h}$, the ratio was low (Fig. 1A and $\mathrm{C}$ ). The lipid droplet formation dramatically increased after $12 \mathrm{~h}$ of the co-culture of Sertoli and germ cells and reached a plateau at $24 \mathrm{~h}$ (Fig. 1B and C). In contrast, the ratio in single-cultured Sertoli cells remained a low level in all time points and showed a slight decrease as the culture continued. Furthermore, the fact that apoptotic spermatogenic cells were not stained by ORO (Fig. 1D) excluded that the lipid droplets were from the germ cells adhered to the Sertoli cells. Taken together, we speculated that the formation of lipid droplets in Sertoli cells in vitro resulted from the phagocytosis of apoptotic spermatogenic cells.

\section{Comparison of lipid droplet formation in Sertoli cells and macrophages after phagocytosis of different apoptotic cells}

It is well-known that macrophages and Sertoli cells can phagocytose apoptotic thymocytes and apoptotic germ cells respectively. In this study, using immunofluorescence-labeled apoptotic cells, we confirmed that the apoptotic spermatogenic cells could also be phagocytosed by macrophages and Sertoli cells (data not shown). However, bacteria (Escherichia coli) or yeast (Saccharomyces cerevisiae) inactivated by heating, common agents used to evaluate phagocytotic activity of macrophages, were not phagocytosed by Sertoli cells (not shown), suggesting a difference of Sertoli cells and macrophages in their phagocytic targets.

In order to analyze whether the formation of lipid droplets after phagocytosis was a cell-type (phagocytic and apoptotic cells) specific phenomenon or a common phenomenon, a comparison study on the lipid droplet formation in the Sertoli cells that phagocytosed apoptotic spermatogenic cells or thymocytes and in the macrophages that phagocytosed apoptotic spermatogenic cells or thymocytes was carried out respectively (Fig. 2A). Abundant lipid droplets were observed in both Sertoli cells and macrophages after phagocytosis of apoptotic spermatogenic cells, although the size of the lipid droplets showed a significant difference in these two kinds of phagocytes (Fig. 2B, lanes 1 and 3). By contrast, only a few lipid droplets were seen in the Sertoli cells and macrophages co-cultured with the apoptotic thymocytes (Fig. 2B, lanes 2 and 4). A timing of lipid droplet formation in Sertoli cells and macrophages co-cultured with apoptotic spermatogenic cells was examined. Abundant lipid droplets appeared in the macrophages as early as $6 \mathrm{~h}$ after co-culture with germ cells (Fig. 2B, lane 3 and Fig. 3), whereas, we did not 
A
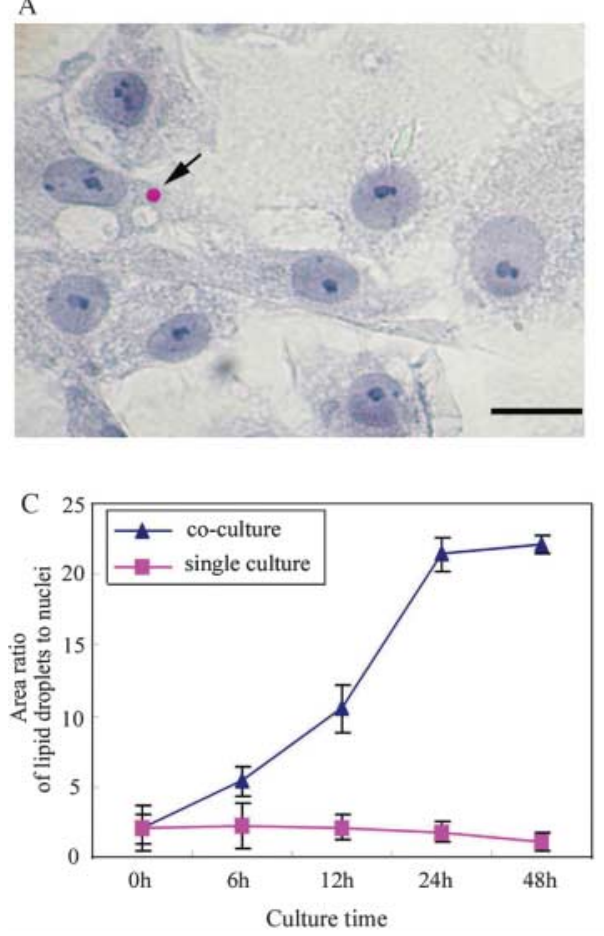

B

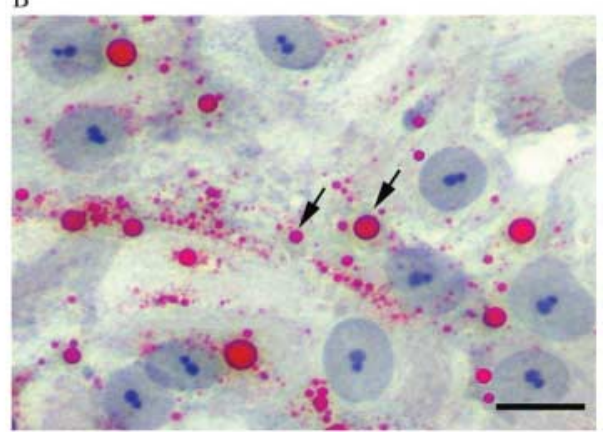

D

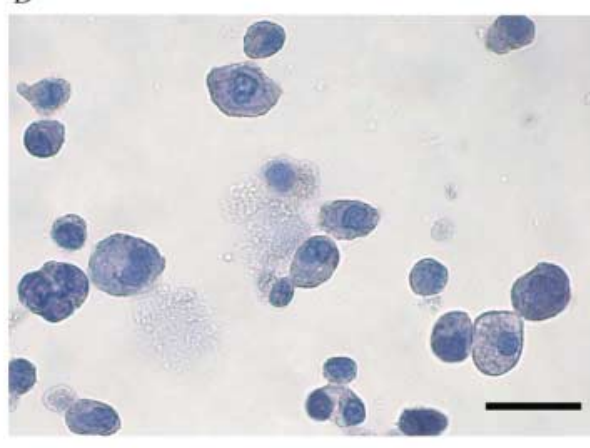

Figure 1 Formation of lipid droplets after phagocytosis of apoptotic germ cells by Sertoli cells. Co-culture of Sertoli and apoptotic germ cells and single-culture of Sertoli cells were performed synchronously. Lipid droplet formation was detected at $0,6,12$, 24 , and $48 \mathrm{~h}$ during the culture by ORO staining. (A) Only a few lipid droplets (arrow) were observed at $0 \mathrm{~h}$. (B) Abundant lipid droplets (arrow) were observed at $24 \mathrm{~h}$. (C) Area ratios of lipid droplets to nucleus in co-culture of Sertoli and spermatogenic cells $(-\mathbf{\Delta}-)$ and single-culture of Sertoli cells (-口-) at different time points were measured. Each datum was from the calculation of 100 cells in three repeat wells. (D) No lipid droplet was observed in degenerating germ cells by ORO staining. Bar $=20 \mu \mathrm{m}$. observe abundant lipid droplets in the Sertoli cells until $12 \mathrm{~h}$ after co-culture with apoptotic germ cells (Fig. 2B, lane 1 and Fig. 3). This result might reflect more phagocytic activities of the macrophages than those of the Sertoli cells and difference in the rates of processing of phagocytosed components in the two cell types.

Although the number of lipid droplets in both Sertoli cells and macrophages co-cultured with the apoptotic spermatogenic cells did not show a significant difference at $24 \mathrm{~h}$ (Fig. 3A), the size of lipid droplets was greatly varied in these two kinds of phagocytes. We divided the lipid droplets into three types based on their diameter under light microscope (magnification $\times 400$ ): large ( $\geq 3 \mu \mathrm{m}$ in diameter), medium $(1-3 \mu \mathrm{m})$, and small $(\leq 1 \mu \mathrm{m})$. As shown in Fig. 2B, we could see many large lipid droplets in the Sertoli cells (lane 1), but a few in the macrophages (lane 3). The comparison of the percentage of the three types of lipid droplets in Sertoli cells and macrophages at $24 \mathrm{~h}$ after co-culture with apoptotic spermatogenic cells was shown in Fig. 3B. Most of the lipid droplets in the macrophages were detected as small. Whereas in the Sertoli cells, about $16.7 \%$ lipid droplets were large, and $26.2 \%$ were medium. This result might reflect different ways of lipid metabolism in Sertoli cells and macrophages. Differences in cell size between Sertoli cells and macrophages might also create physical constraints that influence the size and number of droplets per cell that could be accommodated in the respective cytoplasms.

\section{Phagocytic ability of Sertoli cells in different developing stages postnatal}

It was expected that the different functions would be expressed by mature adult Sertoli cells when compared with prepuberty immature, proliferating Sertoli cells, but there is a surprising lack of definitive comparative studies in the literature. Using ORO staining in this study, we analyzed the lipid-droplet formation in Sertoli cells isolated from mice of different ages with an effort to evaluate the phagocytic capacity of Sertoli cells in different developing stages postnatally. To this aim, the Sertoli cells were isolated from the testes of 1-, 3-, 5-, and 8 -week-old mice and co-cultured with apoptotic spermatogenic cells from 3-week-old mice. At $24 \mathrm{~h}$ after co-culture, the Sertoli cells were stained using ORO, and the lipid droplets were analyzed. To our surprise, almost equal amount of lipid droplets was detected in the Sertoli cells from mice at different postnatal ages after phagocytosis of apoptotic germ cells (Fig. 4), suggesting a similar phagocytic capacity of Sertoli cells at different postnatal stages of development.

\section{Discussion}

Spermatogenesis is a complex process, in which phagocytic clearance of apoptotic spermatogenic cells and residual bodies by Sertoli cell is necessary for healthy spermatogenic cells to proceed through spermatogenesis, although the mechanism remains to be 
A
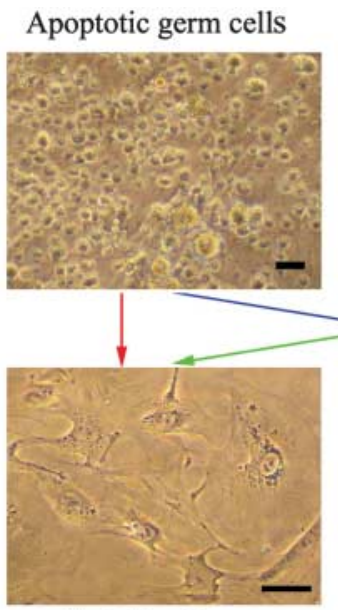

Sertoli cells

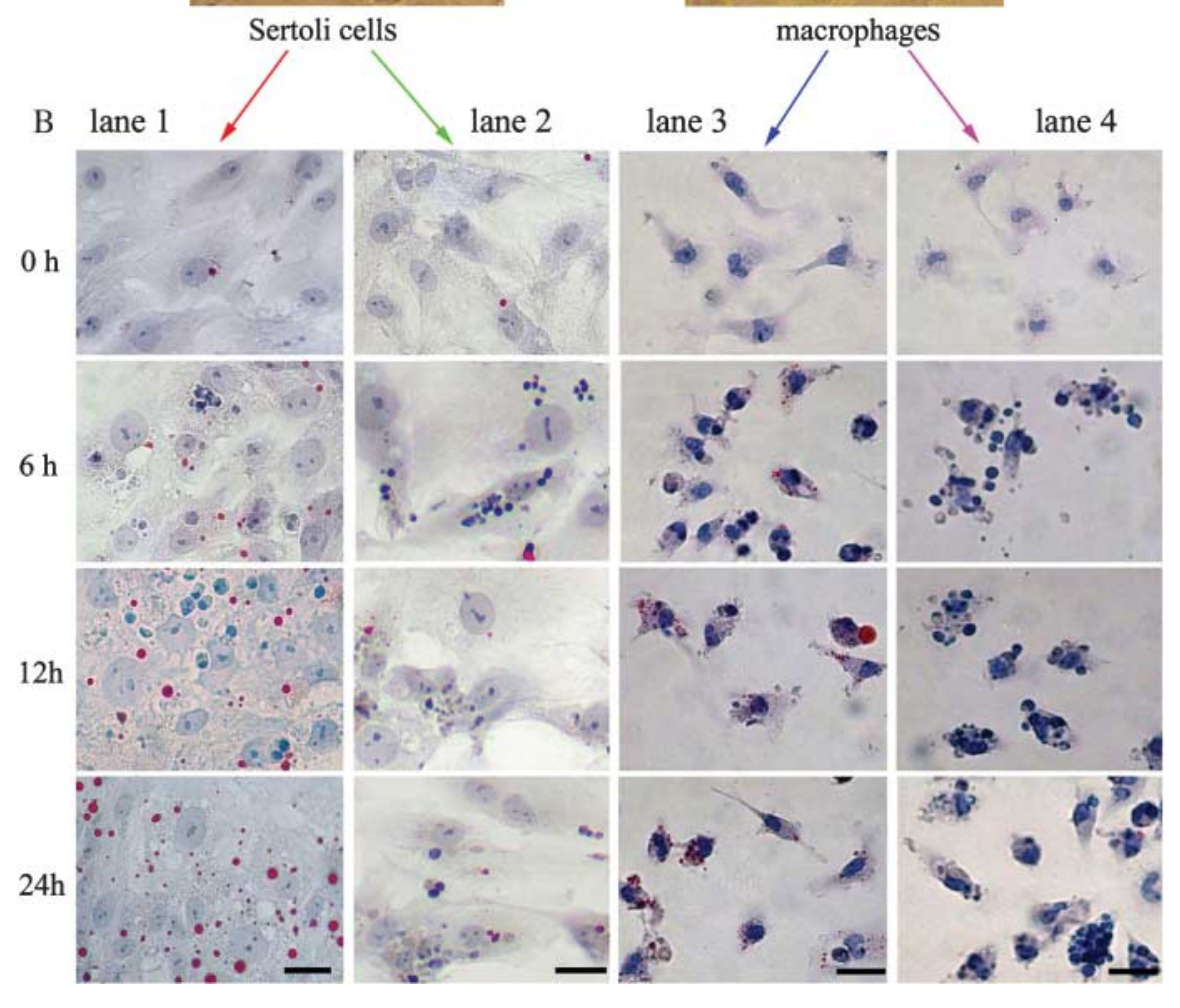

B

lane 1
Apoptotic thymocytes
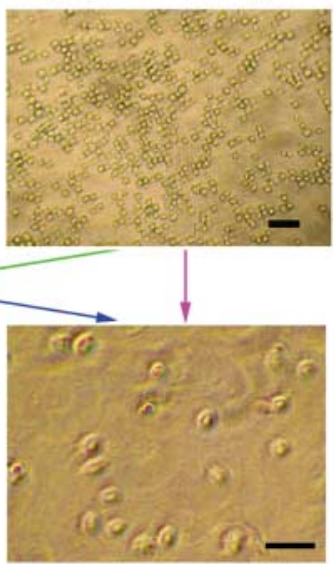

macrophages
Figure 2 Comparison of lipid droplet formation in Sertoli cells and macrophages after phagocytosing apoptotic germ cells and thymocytes. (A) Designs of the experiment and the characteristics of cells. (B) Lipid droplets were stained by ORO in the Sertoli cells after co-culture with apoptotic germ cells (lane 1) and apoptotic thymocytes (lane 2), and in the macrophages after co-culture with apoptotic germ cells (lane 3 ) and apoptotic thymocytes (lane 4). At 0 , $6,12,24 \mathrm{~h}$ during co-culture, apoptotic cells were removed and phagocytes were stained by ORO and hematoxylin. Abundant lipid droplets were observed at $6 \mathrm{~h}$ after co-culture of macrophages and apoptotic germ cells and $12 \mathrm{~h}$ after co-culture of Sertoli and apoptotic germ cells. However, only a few lipid droplets appeared in Sertoli cells and macrophages after co-culture with apoptotic thymocytes at any time points. $\mathrm{Bar}=20 \mu \mathrm{m}$. clarified. The methods for in vitro investigation of the phagocytosis of apoptotic spermatogenic cells by Sertoli cells face a problem that it is complicated to discriminate the germ cells adhered to Sertoli cells from that phagocytosed by Sertoli cells. In this study, we created a practical method of detecting lipid droplets in Sertoli cells by ORO staining to analyze the phagocytosis of apoptotic germ cells by Sertoli cells in vitro.

Previous studies on testis sections by electron microscope disclosed that stage-dependent formation of lipid droplets in Sertoli cells was associated with phagocytosis of residual bodies (Kerr \& de Kretser 1974, Chemes 1986, Ueno \& Mori 1990, Jegou et al. 1991) and apoptotic germ cells (Braun 1998, Pentikainen et al.
2003, Sinha Hikim et al. 2003). The occurrence of spermatogenic cell apoptosis at various stages of differentiation has been reported (Allan et al. 1992, Shikone et al. 1994, Brinkworth et al. 1995, Callard et al. 1995), but only a limited number of apoptotic spermatogenic cells were detected when testis sections were examined histochemically. This was probably due to rapid elimination of apoptotic cells by phagocytosis. Ultrastructure studies with rodent testis sections showed that Sertoli cells could phagocytose degenerating spermatogenic cells (Russell \& Clermont 1977, Chemes 1986, Pineau et al. 1991, Miething 1992, Kerr et al. 1993). Studies in vitro also confirmed that rat Sertoli cell in primary cultures could phagocytose apoptotic 

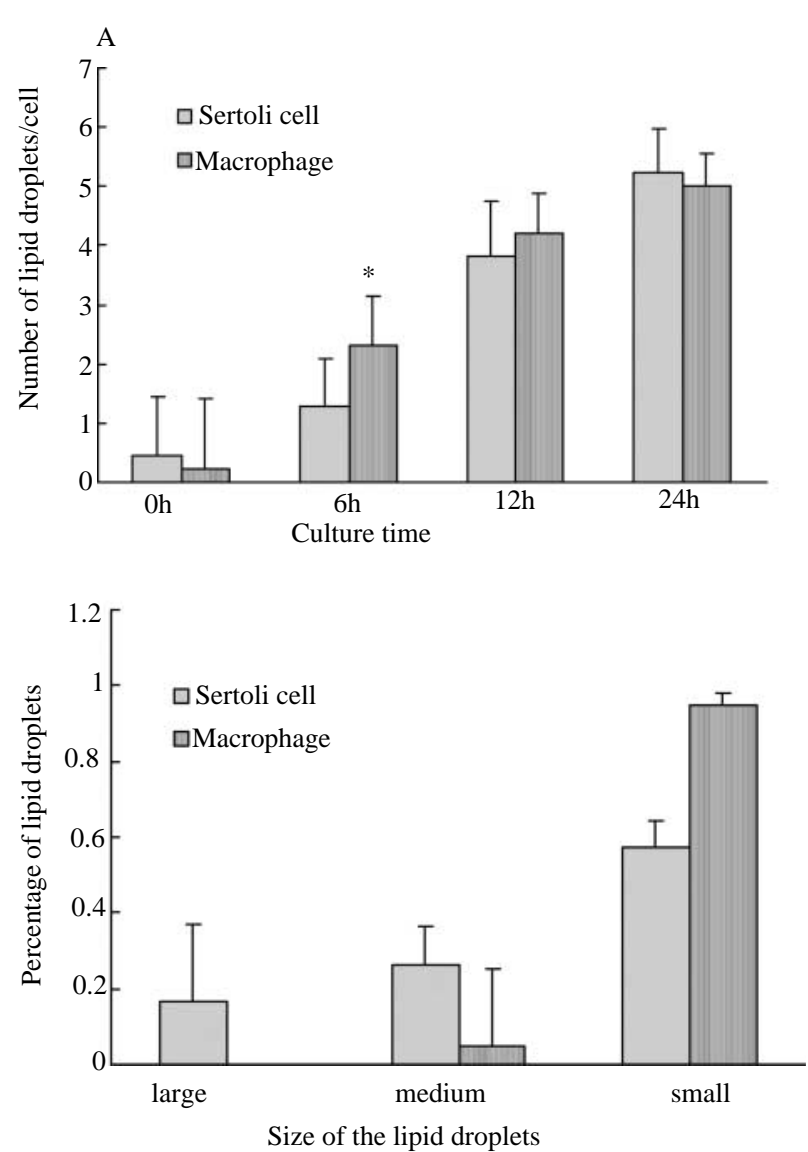

Figure 3 The number (A) and size (B) of lipid droplets in Sertoli cells and macrophages after phagocytosis of apoptotic spermatogenic cells. (A) The lipid droplet numbers at $6 \mathrm{~h}$ after co-culture of macrophages and apoptotic spermatogenic cells were higher than that after co-culture of Sertoli and apoptotic spermatogenic cells $(P<0.01)$. A similar numbers of the lipid droplets at 12 and $24 \mathrm{~h}$ of co-culture were shown in two phagocytes. (B) The size of the lipid droplets showed a significant difference in the Sertoli cells and macrophages. In Sertoli cells, $16.7 \%$ of the lipid droplets were large ( $\geq 3 \mu \mathrm{m}$ in diameter) and $26.2 \%$ of them were medium (1-3 $\mu \mathrm{m})$. In contrast, almost no large and a few medium lipid droplets were observed in macrophages, and $95 \%$ of the lipid droplets in macrophages belonged to small size $(\leq 1 \mu \mathrm{m})$. Each datum was from the calculation of 100 cells in three repeat wells.

spermatogenic cells (Shiratsuchi et al. 1997, 1999). Considering these previous observations, we might conclude that the formation of lipid droplets in our present study was a result of phagocytosis of spermatogenic cells. Detection of lipid droplet formation by ORO staining could be a practical method to analyze phagocytosis of apoptotic germ cells by Sertoli cells in vitro. Adhesion problem in the evaluation on phagocytic function of Sertoli cells in previous studies could be overcome by this approach. It should be noted that a quantitative relationship between measures of phagocytic activity and amount of lipid droplets has not been established in this system.

Various kinds of phagocytic cells can engulf different target cells in different tissues. We asked whether the

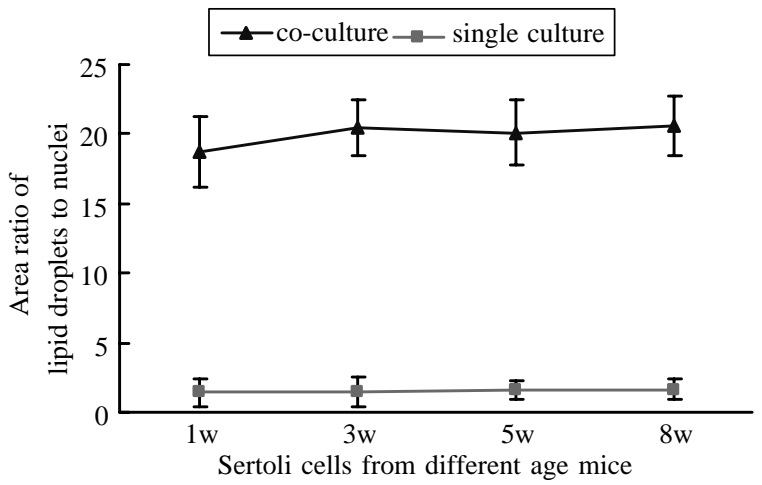

Figure 4 Comparison of lipid droplet formation in Sertoli cells at different postnatal stages of development. Sertoli cells isolated from 1, 3,5 , and 8-week-old mice postnatal were co-cultured with apoptotic spermatogenic cells from 3-week-old mice. After $24 \mathrm{~h}$, spermatogenic cells were removed and Sertoli cells were stained by ORO and hematoxylin. At each time point, single-cultured Sertoli cells were stained as a control. Area ratios of lipid droplets to nucleus in Sertoli cells were measured. Almost equal amount of lipid droplets were showed at different stages of Sertoli cells at $24 \mathrm{~h}$ after co-cultured with apoptotic germ cells. Each datum was from the calculation of 100 cells in three repeat wells.

formation of lipid droplets could be a common criterion for the evaluation of phagocytosis by other phagocytes. Then we tried to answer the question using macrophages, a well-known phagocytic cell type widely existed in many kinds of tissue and immune system (Ellis et al. 1991, Francavilla et al. 2002). The results demonstrated that the apoptotic cell-type phagocytosed by phagocytes was related to the formation of lipid droplets. We proposed that the internal membrane system of apoptotic cells determined the formation of lipid droplets. Compared to thymocytes, spermatogenic cells might contain more internal membranes, including rich lipids, such as mitochondria and Golgi apparatus. The mechanism of lipid droplet formation after phagocytosis has been explained in two different ways. Kerr et al. (1984) thought that an accumulation of lipid droplets after phagocytosis of residual bodies represented the resynthesis of lipids by Sertoli cells, whereas Chemes (1986) proposed that the lipid droplets were last remnants of digested residual bodies. This in vitro system may provide a practical approach to confirm the way of the lipid droplet formation by blocking the pathway to synthesize lipid in Sertoli cells.

A previous study showed that Sertoli cells from 20-dayold rats in vitro were capable of phagocytosing all types of spermatogenic cells undergoing apoptosis during spermatogenesis (Shiratsuchi et al. 1999). During postnatal development of testis, Sertoli cells undergo a radical switch from an immature, proliferative state to a mature, non-proliferative state at around the onset of puberty (Sharpe et al. 2003). Adult Sertoli cells perform dual phagocytic functions, namely phagocytosing apoptotic spermatogenic cells and residual bodies from elongated spermatids. A comparison study on the phagocytic ability 
of Sertoli cells in different development stages has not been reported. Our present study demonstrates for the first time that Sertoli cells from different age mice have a similar ability in the phagocytosis of apoptotic spermatogenic cells. Lipid droplets were first reported as the Sertoli cell component by the original discoverer of the cell (Sertoli 1865), the amount of the lipid droplets varies considerably between stages of spermatogenesis, as well as between species. It has been hypothesized that lipid droplets in Sertoli cell are evidence to recycle lipids from the residual bodies and degenerated germ cells (Russell 1993). This study provided a direct evidence to support this hypothesis.

Accumulated lipid droplets in Sertoli cells can be used for nourishing germ cells. It has been reported that Sertoli cell lipids were absorbed into spermatids during certain stages of spermatogenesis, in which the spermatids are embedded in Sertoli cell cytoplasm (Lynch \& Scott 1951, Lacy 1962). Other studies reported a transfer of these lipid inclusions from the Sertoli cells to primary spermatocytes (Kerr \& De Kretser 1975). Several recent studies showed that the accumulation of excess lipid droplets in Sertoli cells resulted in impaired spermatogenesis in mice deficient in genes related to lipid metabolism or transfer such as the nuclear receptors, retinoid $X$ receptor- $\beta$ (RXR $\beta$ ) (Mascrez et al. 2004), liver $X$ receptor- $\beta$ (LXR $\beta$ ) (Robertson et al. 2005), transcriptional intermediary factor $2 /$ glucocorticoid receptor-interacting protein 1 (TIF2/GRIP1), a nuclear receptor co-activator (Gehin et al. 2002), Cnot7, a regulator of RXRß (Nakamura et al. 2004), ATP-binding cassette transporter 1 (ABCA1), a transporter that shuttled excess cholesterol and phospholipids out of the cells (Selva et al. 2004), and multifunctional protein 2 (MFP-2), which was associated with peroxisomal $\beta$-oxidation (Huyghe et al. 2006). Thus, a balance of the metabolism of lipids in Sertoli cell is critical to maintain normal spermatogenesis. The question of lipid metabolism in this cell type remains open. The in vitro model in this study could be useful to investigate the cycle of lipid formation in Sertoli cells and lipid function in regulating spermatogenesis.

In summary, a practical in vitro method has been developed to investigate phagocytic function of Sertoli cells through detecting the lipid droplet formation by ORO staining. Using this approach, we demonstrated that the lipid droplet formation depended on the apoptotic cell type, but not the phagocyte type. The results of the present study showed a similar phagocytic ability of the Sertoli cells at different developing stages postnatally. This approach could also be a practical model to study the lipid formation, metabolism, and function in Sertoli cells.

\section{Acknowledgements}

This work was supported by the National Natural Science Foundation of China (Grant nos 30470878 and 30570678). The authors declare that there is no conflict of interest that would prejudice the impartiality of this scientific work.

\section{References}

Allan DJ, Harmon BV \& Roberts SA 1992 Spermatogonial apoptosis has three morphologically recognizable phases and shows no circadian rhythm during normal spermatogenesis in the rat. Cell Proliferation 25 241-250.

Blanco-Rodriguez J \& Martinez-Garcia C 1996 Spontaneous germ cell death in the testis of the adult rat takes the form of apoptosis: re-evaluation of cell types that exhibit the ability to die during spermatogenesis. Cell Proliferation 29 13-31.

Braun RE 1998 Every sperm is sacred - or is it? Nature Genetics 18 202-204.

Brinkworth MH, Weinbauer GF, Schlatt S \& Nieschlag E 1995 Identification of male germ cells undergoing apoptosis in adult rats. Journal of Reproduction and Fertility 105 25-33.

Callard GV, Jorgensen JC \& Redding JM 1995 Biochemical analysis of programmed cell death during premeiotic stages of spermatogenesis in vivo and in vitro. Developmental Genetics 16 140-147.

Chemes H 1986 The phagocytic function of Sertoli cells: a morphological, biochemical, and endocrinological study of lysosomes and acid phosphatase localization in the rat testis. Endocrinology 119 1673-1681.

Cheng CY, Mather JP, Byer AL \& Bardin CW 1986 Identification of hormonally responsive proteins in primary Sertoli cell culture medium by anion-exchange high performance liquid chromatography. Endocrinology 118 480-488.

Chong MMW, Metcalf D, Jamieson E, Alexander WS \& Kay TWH 2005 Suppressor of cytokine signaling- 1 in T cells and macrophages is critical for preventing lethal inflammation. Blood 106 1668-1675.

Dym M 1994 Spermatogonial stem cells of the testis. PNAS 91 11287-11289.

Ellis RE, Yuan J \& Horvitz HR 1991 Mechanisms and functions of cell death. Annual Reviews of Cell Biology 7 663-698.

Francavilla S, D'Abrizio P, Cordenchi G, Pelliccione F, Necozione S, Ulisse S, Properzi G \& Francavilla F 2002 Fas expression correlates with human germ cell degeneration in meiotic and post-meiotic arrest of spermatogenesis. Molecular Human Reproduction 8 213-220.

Gehin M, Mark M, Dennefeld C, Dierich A, Gronemeyer H \& Chambon P 2002 The function of TIF2/GRIP1 in mouse reproduction is distinct from those of SRC-1 and p/CIP. Molecular Cell Biology 22 5923-5937.

Huckins C 1978 The morphology and kinetics of spermatogonial degeneration in normal adult rats: an analysis using a simplified classification of germinal epithelium. The Anatomical Record 190 905-926.

Huyghe S, Schmalbruch H, De Gendt K, Verhoeven G, Guillou F, Van Veldhoven PP \& Baes M 2006 Peroxisomal multifunctional protein 2 is essential for lipid homeostasis in Sertoli cells and male fertility in mice. Endocrinology 147 2228-2236.

Jegou B 1991 Spermatids are regulators of Sertoli cell function. Annals of the New York Academy of Sciences 637 340-353.

Johnson L, Petty CS \& Neaves WB 1983 Further quantification of human spermatogenesis: germ cell loss during post-prophase of meiosis and its relationship to daily sperm production. Biology of Reproduction 29 207-215.

Kerr JB \& de Kretser DM 1974 Proceedings: the role of Sertoli cell in phagocytosis of the residual bodies of spermatids. Journal of Reproduction and Fertility 36 439-440.

Kerr JB \& de Kretser DM 1975 Cyclic variations in Sertoli cell lipid content throughout the spermatogenic cycle in the rat. Journal of Reproduction and Fertility 43 1-8.

Kerr JB, Mayberry RA \& Irby DC 1984 Morphometric studies on lipid inclusions in Sertoli cells during the spermatogenic cycle in the rat. Cell and Tissue Research 236 699-709.

Kerr JB, Millar M, Maddocks S \& Sharpe RM 1993 Stage-dependent changes in spermatogenesis and Sertoli cells in relation to the onset of spermatogenic failure following withdrawal of testosterone. The Anatomical Record 235 547-559. 
Lacy D 1962 Certain aspects of testis structure and function. British Medical Bulletin 18 205-208.

Lillie RD \& Fullmer HM 1976 Histopathologic Technic and Practical Histochemistry, 4th edn New York: Mc-Graw Hill, pp 559-600.

Lynch KM \& Scott WW 1951 Lipid distribution in the Sertoli cell and Leydig cell of the rat testis as related to experimental alterations of the pituitary-gonad system. Endocrinology 49 8-14.

Mascrez B, Ghyselinck NB, Watanabe M, Annicotte J-S, Chambon P, Auwerx J \& Mark M 2004 Ligand-dependent contribution of RXR $\beta$ to cholesterol homeostasis in Sertoli cells. EMBO Journal 5 285-290.

Miething A 1992 Germ-cell death during prespermatogenesis in the testis of the golden hamster. Cell and Tissue Research 267 583-590.

Nakamura T, Yao R, Ogawa T, Suzuki T, Ito C, Tsunekawa N, Inoue K, Ajima R, Miyasaka T, Yoshida Y, Ogura A, Toshimori K, Noce T, Yamamoto T \& Noda T 2004 Oligo-asthenoteratozoospermiain mice lacking Cnot7, a regulator of retinoid $\mathrm{X}$ receptor beta. Nature Genetics 36 528-533.

Oakberg EF 1956 A description of spermatogenesis in the mouse and its use in analysis of the cycle of seminiferous epithelium and germ cell renewal. American Journal of Anatomy 99 391-413.

Packer Al, Besmer P \& Bachvarova RF 1995 Kit ligand mediates survival of type A spermatogonia and dividing spermatocytes in postnatal mouse testes. Molecular Reproduction and Development 42 303-310.

Pentikainen V, Dunkel L \& Erkkila K 2003 Male germ cell apoptosis. Endocrine Development 5 56-80.

Pineau C, Le Magueresse B, Courtens JL \& Jegou B 1991 Study in vitro of the phagocytic function of Sertoli cells in the rat. Cell and Tissue Research 264 589-598.

Ren Y \& Savill J 1998 Apoptosis: the importance of being eaten. Cell Death and Differentiation 5 563-568.

Robertson KM, Schuster GU, Steffensen KR, Hovatta O, Meaney S, Hultenby K, Johansson LC, Svechnikov K, Soder O \& Gustafsson JA 2005 The liver $X$ receptor- $\beta$ is essential for maintaining cholesterol homeostasis in the testis. Endocrinology 146 2519-2530.

Russell LD 1993 Form, dimensions, and cytology of mammalian Sertoli cells. In The Sertoli Cell, pp 1-37. Eds LD Russell \& MD Griswold. Clearwater: Cache River Press.

Russell LD \& Clermont Y 1977 Degeneration of germ cells in normal, hypophysectomized and hormone treated hypophysectomized rats. The Anatomical Record 187 347-366.
Scott RS, McMahon EJ, Pop SM, Reap EA, Caricchio R, Cohen PL, Earp HS \& Matsushima GK 2001 Phagocytosis and clearance of apoptotic cells is mediated by Mer. Nature 411 207-211.

Selva DM, Hirsch-Reinshagen V, Burgess B, Zhou S, Chan J, Mclsaac S, Hayden MR, Hammond GL, Vogl AW \& Wellington CL 2004 The ATP-binding cassette transporter 1 mediates lipid efflux from Sertoli cells and influences male fertility. Journal of Lipid Research 45 1040-1050.

Sertoli E 1865 De l'esistenza di particulari cellule ramificate nei canalicoli seminiferi del testicolo umano. Morgagni 7 31-40.

Sharpe RM, McKinnell C, Kivlin C \& Fisher JS 2003 Proliferation and functional maturation of Sertoli cells, and their relevance to testis function in adulthood. Reproduction 125 769-789.

Shikone T, Billig H \& Hsueh AJW 1994 Experimentally induced cryptorchidism increases apoptosis in rat testis. Biology of Reproduction 51 865-872.

Shiratsuchi A, Umeda M, Ohba Y \& Nakanishi Y 1997 Recognition of phosphatidylserine on the surface of apoptotic spermatogenic cells and subsequent phagocytosis by Sertoli cells of the rat. Journal of Biological Chemistry 272 2354-2358.

Shiratsuchi A, Kawasaki Y, Ikemoto M, Arai H \& Nakanishi Y 1999 Role of class B scavenger receptor type I in phagocytosis of apoptotic rat spermatogenic cells by Sertoli cells. Journal of Biological Chemistry 274 5901-5908.

Sinha Hikim AP, Lue Y, Diaz-Romero M, Yen PH, Wang C \& Swerdloff RS 2003 Deciphering the pathways of germ cell apoptosis in the testis. Journal of Steroid Biochemistry and Molecular Biology 85 175-182.

Ueno H \& Mori H 1990 Morphometrical analysis of Sertoli cell ultrastructure during the seminiferous epithelial cycle in rats. Biology of Reproduction 43 769-776.

Received 4 April 2006

First decision 10 May 2006

Revised manuscript received 22 May 2006

Accepted 16 June 2006 